\title{
Landscape of Simple Superconformal Field Theories in 4D
}

\author{
Kazunobu Maruyoshi, ${ }^{1, *}$ Emily Nardoni, ${ }^{2,3, \dagger}$ and Jaewon Song ${ }^{4, \$}$ \\ ${ }^{1}$ Faculty of Science and Technology, Seikei University, 3-3-1 Kichijoji-Kitamachi, Musashino-shi, Tokyo 180-8633, Japan \\ ${ }^{2}$ Mani L. Bhaumik Institute for Theoretical Physics, Department of Physics and Astronomy, \\ University of California, Los Angeles, California 90095, USA \\ ${ }^{3}$ Department of Physics, University of California, San Diego, La Jolla, California 92093, USA \\ ${ }^{4}$ School of Physics, Korea Institute for Advanced Study, 85 Hoegiro, Dongdaemun-gu, Seoul 02455, Korea
}

(Received 28 November 2018; published 26 March 2019)

\begin{abstract}
We explore the space of renormalization group flows that originate from supersymmetric $\mathcal{N}=1 S U(2)$ gauge theory with one adjoint and a pair of fundamental chiral multiplets. By considering all possible relevant deformations-including coupling to gauge-singlet chiral multiplets-we find 34 fixed points in this simple setup. We observe that theories in this class exhibit many novel phenomena: emergent symmetry, decoupling of operators, and narrow distribution of central charges $a / c$. This set of theories includes two of the $\mathcal{N}=2$ minimal Argyres-Douglas theories and their mass deformed versions. In addition, we find 36 candidate fixed point theories possessing unphysical fermionic operators_-including one with central charges $(a, c) \simeq(0.20,0.22)$ that are smaller than any known superconformal theory-that need further investigation.
\end{abstract}

DOI: 10.1103/PhysRevLett.122.121601

Introduction.-Conformal field theory (CFT) is an important object in theoretical physics which displays the physics of the low energy fixed points of some gauge theories and of critical phenomena in condensed matter theories. One interesting question of CFT is to find the "minimal" interacting theory. In four dimensions, a measure of minimality is the $a$ central charge, the coefficient to the Euler density of the trace anomaly, due to the $a$ theorem [1,2], $a_{\mathrm{UV}}>a_{\mathrm{IR}}$ for all unitary renormalization group (RG) flows. A related quantity is the $c$ central charge, the coefficient to the twopoint function of the stress-energy tensor.

However, it is difficult to analyze strongly coupled CFTs, even $a$ and $c$, in general. The conformal bootstrap program [3] partially solves this by giving a bound on $c$ but does not tell what the actual minimal theory is. The situation changes drastically in theories with supersymmetry. The superconformal symmetry allows us to relate the central charges to 't Hooft anomalies of the $R$ symmetry [4], which are determined by the $a$ maximization [5] or higher supersymmetry itself.

The central charge $c$ of any unitary interacting $\mathcal{N}=2$ superconformal field theory (SCFT) satisfies $c \geq \frac{11}{30}[6]$. The theory that saturates the bound is the Argyres-Douglas theory [7,8], denoted as $H_{0}$ or $\left(A_{1}, A_{2}\right)$ in the literature. $H_{0}$ also has the smallest known value of $a$ for an interacting $\mathcal{N}=2$ theory.

Published by the American Physical Society under the terms of the Creative Commons Attribution 4.0 International license. Further distribution of this work must maintain attribution to the author(s) and the published article's title, journal citation, and DOI. Funded by SCOAP .
In $\mathcal{N}=1$ theories, no analytic bound on the central charges is known so far. However, the numerical bootstrap suggests that the SCFT with the minimal central charge has a chiral operator $O$ with chiral ring relation $O^{2}=0$ [9-11], and a bound $c \geq 1 / 9 \simeq 0.11$ [11]. Is there a theory which saturates this bound? The minimal theory thus far known in the literature has $a=\frac{263}{768} \simeq 0.34$ and $c=\frac{271}{768} \simeq 0.35$, which was constructed via a deformation of the $H_{0}$ theory $[12,13]$, and thus denoted as $H_{0}^{*}$ [14]. This value of $c$ is large compared to the bound.

In the present work, we initiate a classification of $\mathcal{N}=1$ SCFT in four dimensions obtained from Lagrangian theories to find a minimal SCFT. We explore the space of RG flows and fixed points that originate from the simple starting point of supersymmetric $S U(2)$ gauge theory with one adjoint and a pair of fundamental chiral multiplets. From this minimal matter content, we consider all of the possible relevant deformations, including deformations by coupling gaugesinglet chiral multiplets. Among the fixed points that we obtain, two have enhanced $\mathcal{N}=2$ supersymmetry: the Argyres-Douglas theories $H_{0}$ and $H_{1}$, as already found in Refs. [15-17]. The other 32 are $\mathcal{N}=1$ supersymmetric, including the $H_{0}^{*}$ theory as a minimal theory in terms of $a$. We verify that these are "good" theories in the sense that there is no unitary-violating operator by utilizing the superconformal indices $[18,19]$. It is remarkable that $(a, c)$ of these SCFTs distribute within a narrow range as in Fig. 1, although the allowed bound of $a / c$ is wide [20].

In addition, we find 36 candidate fixed points which have an accidental global symmetry in the infrared and some unphysical operators; thus we refer to them as "bad" theories. 
Remarkably, these include theories with even smaller central charges than those of $H_{0}^{*}$. The minimal one, which we denote as $\mathcal{T}_{M}$, has $a \simeq 0.20$ and $c \simeq 0.22$. Although we are not able to conclude that these "bad" theories are really physical by the present techniques, we scope their properties.

A landscape of simple SCFTs. - We systematically enumerate a large set of superconformal fixed points via the following procedure:

(1) Start with some fixed point theory $\mathcal{T}$.

(2) Find the set of all of the relevant chiral operators of $\mathcal{T}$, which we will call $\mathcal{R}_{\mathcal{T}}$. Let us also denote $\mathcal{S}_{\mathcal{T}} \subset \mathcal{R}_{\mathcal{T}}$ as the set of operators with $R$ charge less than $4 / 3$.

(3) Consider the fixed points $\left\{\mathcal{T}_{O}\right\}$ obtained by the deformation $\delta W=O$ for all $O \in \mathcal{R}_{\mathcal{T}}$.

(4) Consider the fixed points $\left\{\mathcal{T}_{\bar{O}}\right\}$ given by adding an additional gauge-singlet $\mathcal{N}=1$ chiral field $M$ and the superpotential coupling $\delta W=M O$ for all $O \in \mathcal{S}_{\mathcal{T}}$.

(5) For each of the new fixed point theories obtained in previous steps, check to see whether it has an operator $O_{d}$ that decouples. Remove it by introducing an $\mathcal{N}=1$ chiral field $X$ and a superpotential coupling $\delta W=X O_{d}$. We will use this notation to clearly distinguish $X$ from $M$ in the following.

(6) For each new fixed point, repeat the entire procedure. Terminate if there is no new fixed point.

We employ the $a$-maximization procedure [5] and its modification [21] to compute the superconformal $R$ charges at each step. Beyond $a$ maximization, we check to see whether the theory passes basic tests as a viable unitary SCFT: one is the Hofman-Maldacena bounds for $\mathcal{N}=1$ SCFTs, $\frac{1}{2} \leq(a / c) \leq \frac{3}{2}$ [20]; the other one is the superconformal index. Some of the candidate fixed points have trivial index or violate the unitarity constraints $[22,23]$.

We perform this procedure for $S U(2)$ gauge theory with the adjoint chiral multiplet $\phi$ and two fundamental chiral multiplets, $q$ and $\tilde{q}\left(N_{f}=1\right)$. When there is no superpotential, this theory flows to an interacting SCFT $\hat{\mathcal{T}}$, as discussed in Ref. [24] (also see Ref. [25]), and a free chiral multiplet $\operatorname{Tr} \phi^{2}$. To pick up only the interacting piece, we add the additional singlet $X$ and the superpotential $W_{\hat{\mathcal{T}}}=X \operatorname{Tr} \phi^{2}$.

Starting from $\hat{\mathcal{T}}$, we apply the deformation procedure and find 34 nontrivial distinct fixed points. These theories pass every test we have checked, so we call them "good" theories. One caveat is that some of these "good" theories have a flavor symmetry that is not classically manifest. This can be explicitly seen from the superconformal index, as we will discuss shortly. In these cases, we cannot rule out the possibility that this symmetry mixes with the superconformal $R$ symmetry causing unitarity violation. Nevertheless we keep referring to these theories as good, assuming that there is no such mixing. We list the theories with this feature in the Supplemental Material [26].

There are an additional 36 distinct theories that pass almost all of our checks, except that there is a term in the index that signals a violation of unitarity. The existence of

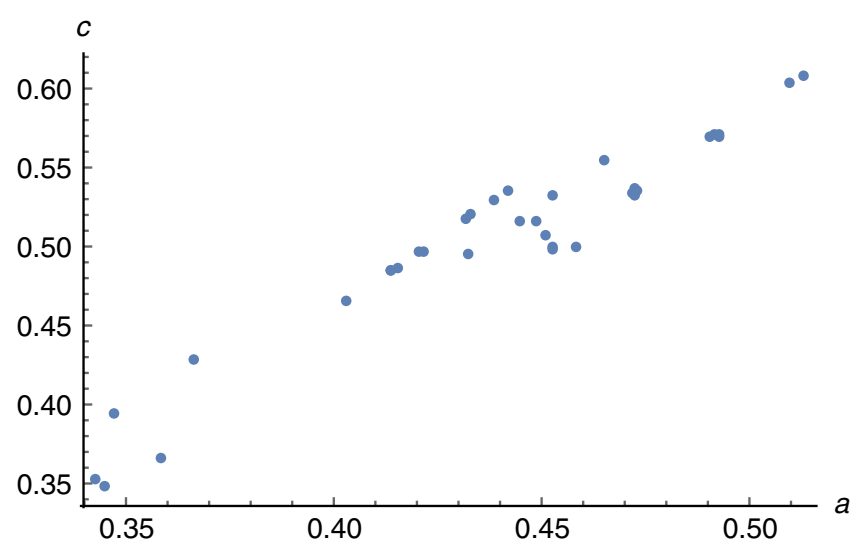

FIG. 1. The central charges of the 34 "good" theories. The ratios $a / c$ all lie within the range $(0.8246,0.9895)$. The mean value of $a / c$ is 0.8732 , with standard deviation 0.0403 .

such a term implies that either the theory does not flow to a SCFT in the IR or the answers we obtained were incorrect because we failed to take into account an accidental symmetry. In fact, these "bad" theories also have an accidental $U(1)$ symmetry which is not visible at the level of the superpotential but is evident by the existence of the corresponding conserved current term present in the index. At present we do not know how to account for this accidental symmetry, so we cannot say for certain whether or not these flows will lead to SCFTs.

Interestingly, six of these "bad" theories appear to have central charges lower than that of $H_{0}^{*}$. Denote the lowest one $\mathcal{T}_{M}$. This is a hint that there might be a minimal SCFT in this landscape.

We have plotted $a, c$ for the "good" theories without this interesting complication in Fig. 1. We see that the distribution of $a$ vs $c$ is concentrated near the line of $a / c \sim 0.87$. All of the theories satisfy the Hofman-Maldacena bound, and more curiously the stronger bound conjectured in Ref. [27], $\frac{3}{5} \leq(a / c)$. Of the "good" theories, $H_{0}^{*}$ has the smallest value of $a . \mathcal{T}_{0}$ has the smallest value of $a$ among any theory with a $U(1)$ flavor symmetry. $H_{1}^{*}$ has the smallest value of $a$ among any theory with an $S U(2)$ flavor symmetry [28]. Below we examine each of these minimal theories in turn, as well as the lowest central charge theory $\mathcal{T}_{M}$, and the second-to-lowest $a$ central charge "good" theory with no flavor symmetry, which we denote $\mathcal{T}_{\mu}$. We summarize the structure of RG flows among these special theories in Fig. 2. The full list of our theories appears in the Supplemental Material [26].

The superconformal indices of these theories can be computed using the Lagrangian description. We define the index as

$$
\mathcal{I}(t, y ; x)=\operatorname{Tr}(-1)^{F} t^{3\left(r+2 j_{1}\right)} y^{2 j_{2}} x^{f},
$$

where $\left(j_{1}, j_{2}\right)$ are the spins of the Lorentz group and $r$ the $U(1) R$ charge. When the theory has a global symmetry with Cartan generator $f$, we also include the fugacity $x$ for 


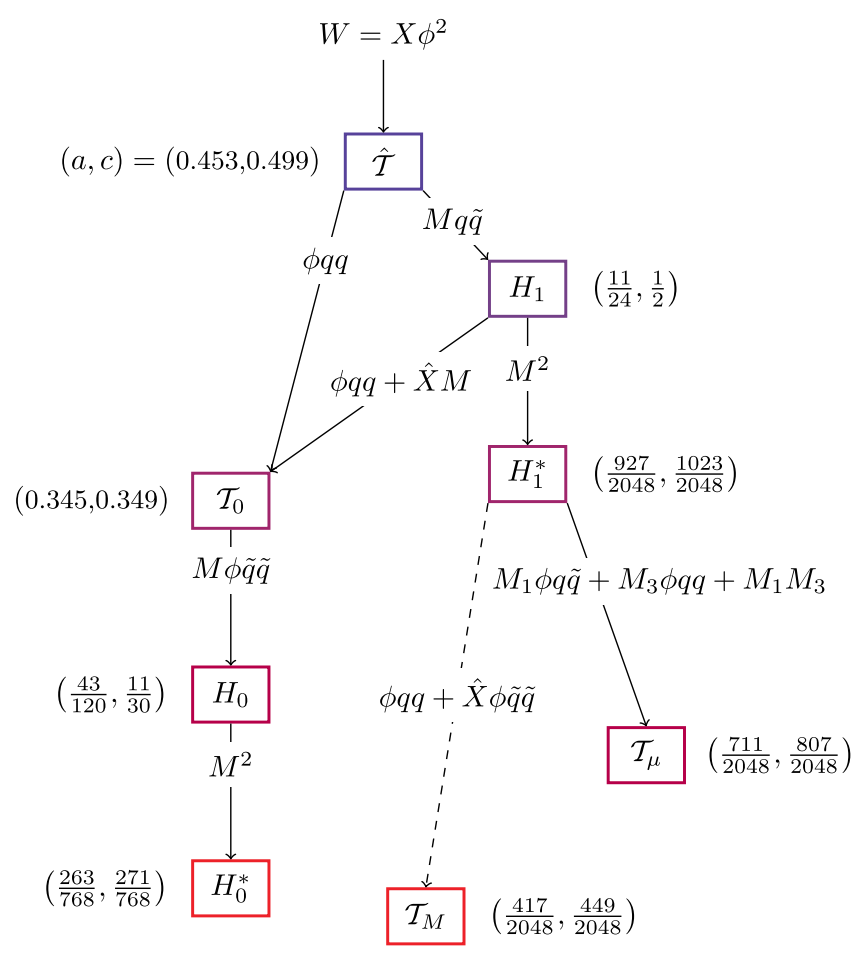

FIG. 2. A subset of the fixed points that can be obtained from $S U(2) N_{f}=1$ adjoint SQCD with singlets. The arrows are labeled with the superpotential deformations. Note that the graph is not arranged vertically by decreasing the $a$ central charge because the deformations we consider involve coupling in the singlet fields.

it. For each of these special theories, we give the first few terms in the reduced superconformal index

$$
\mathcal{I}_{r}(t, y)=\left(1-t^{3} / y\right)\left(1-t^{3} y\right)[\mathcal{I}(t, y)-1],
$$

which removes the conformal descendant contributions coming from spacetime derivatives. If the reduced index contains a term $t^{R} \chi_{2 j+1}(y)$ with $R<2+2 j$ or a term $(-1)^{2 j+1} t^{R} \chi_{2 j+1}(y)$ with $2+2 j \leq R<6+2 j$, it violates the unitarity constraint $[22,23]$.

The coefficient of $t^{6} y^{0}$ allows us to read off the number of marginal operators minus the number of conserved currents [22]. The superpotential $F$ terms $\partial W / \partial \varphi=0$ for the fields $\varphi$ allow us to read off the classical chiral ring, and quantum modifications can be argued from the index. We will see that the chiral rings we study in this Letter are subject to quantum corrections. The superconformal index turns out to be a useful tool to study the fully quantum corrected chiral rings of our models.

$\mathcal{T}_{0}$ : Minimal $c$, minimal a with $U(1)$. - Let us begin with the $\mathcal{T}_{0}$ SCFT which is obtained via a deformation of $\hat{\mathcal{T}}$,

$$
W_{\mathcal{T}_{0}}=X \operatorname{Tr} \phi^{2}+\operatorname{Tr} \phi q q
$$

and has irrational central charges

$$
\begin{aligned}
& a_{\mathcal{T}_{0}}=\frac{81108+1465 \sqrt{1465}}{397488} \simeq 0.3451, \\
& c_{\mathcal{T}_{0}}=\frac{29088+1051 \sqrt{1465}}{198744} \simeq 0.3488 .
\end{aligned}
$$

The IR $R$ charges of the fields of the $\mathcal{T}_{0}$ and all other theories discussed below are given in Table I. This theory has the second smallest value of $a$, and the smallest value of $c$ among the 34 good fixed points that we find [29].

The chiral ring of the theory can be easily studied: the $F$ term conditions from Eq. (3) are simply $\operatorname{Tr} \phi^{2}=0, q \phi=0$, and $X \phi+q^{2}=0$. The first equation truncates the chiral ring by setting $\phi^{2}=0$. The second and third equations lead to the classical generators of the chiral ring: $O^{\prime} \equiv \operatorname{Tr} q \tilde{q}$, $\operatorname{Tr} \phi \tilde{q} \tilde{q}$, and $X$, with relation $O^{\prime 2} \sim X \operatorname{Tr} \phi \tilde{q} \tilde{q}$.

This theory has an anomaly-free $U(1)$ flavor symmetry that mixes with $R$. The reduced index is given as

$$
\begin{aligned}
\mathcal{I}_{r}(t, y ; x)= & t^{3.28} x^{12}-t^{3.45} x^{-2} \chi_{2}(y)+t^{4.19} x^{8}-t^{6} \\
& +t^{6.56} x^{24}+t^{7.46} x^{20}+t^{8.27} x^{-10}+\cdots,
\end{aligned}
$$

where we assigned the flavor charges for the fugacity $x$ as $f_{q}=1, f_{\tilde{q}}=7, f_{\phi}=-2, f_{X}=4$. Here and below $\chi_{s}(a)$ denotes the character for the $S U(2)$ flavor symmetry of dimension $s=2 j+1$. This index allows us to read off the quantum modified chiral ring: the terms $t^{3.28} x^{12}$ and $t^{4.19} x^{8}$ in the index come from the chiral operators $\operatorname{Tr} \phi \tilde{q} \tilde{q}$ and $\operatorname{Tr} q \tilde{q}$, respectively; the second term denotes the fermionic

TABLE I. The $R$ charges of the chiral multiplets at various fixed points. The $\mathcal{T}_{\mu}$ theory has three chiral multiplets labeled $M$, which we denote as $M_{1,2,3}$.

\begin{tabular}{lccccc}
\hline \hline Fields & $\mathcal{T}_{0}$ & $H_{0}^{*}$ & $H_{1}^{*}$ & $\mathcal{T}_{\mu}$ & $\mathcal{T}_{M}$ \\
\hline$q$ & $(543-\sqrt{1465} / 546) \simeq 0.924$ & $11 / 12$ & $1 / 2$ & $1 / 4$ & $7 / 8$ \\
$\tilde{q}$ & $(75-\sqrt{1465} / 78) \simeq 0.471$ & $5 / 12$ & $1 / 2$ & $3 / 4$ & $1 / 8$ \\
$\phi$ & $(3+\sqrt{1465} / 273) \simeq 0.151$ & $1 / 6$ & $1 / 4$ & $1 / 4$ & $1 / 4$ \\
$M$ & $\cdots$ & 1 & 1 & $(3 / 4,1,5 / 4)$ & 1 \\
$X$ & {$[2(270-\sqrt{1465}) / 273] \simeq 1.70$} & $5 / 3$ & $3 / 2$ & $3 / 2$ & $3 / 2$ \\
$\hat{X}$ & $\cdots$ & $\cdots$ & $\cdots$ & $\cdots$ & $3 / 2$ \\
\hline \hline
\end{tabular}


operator $O_{\alpha}=\operatorname{Tr} \phi W_{\alpha}$. We see that the operator $X$ (which would contribute $t^{5.10} x^{4}$ to the index if it exists) is absent from the chiral ring [30]. We can read off the chiral ring relation $O^{\prime 2}=O_{\alpha}(\operatorname{Tr} \phi \tilde{q} \tilde{q})=0$ from the absence of the terms $t^{8.38} x^{16}$ and $-t^{6.73} \chi_{2}(y) x^{10}$.

$H_{0}^{*}$ : Minimal a.-The $H_{0}$ fixed point can be obtained from $\mathcal{T}_{0}$ by adding the $M \operatorname{Tr} \phi \tilde{q} \tilde{q}$ term. This superpotential is indeed a simplified version of the one considered in Ref. [15]. At the $H_{0}$ fixed point, we further deform by a mass term $M^{2}$,

$$
W_{H_{0}^{*}}=X \operatorname{Tr} \phi^{2}+\operatorname{Tr} \phi q q+M \operatorname{Tr} \phi \tilde{q} \tilde{q}+M^{2} .
$$

This flows to the $H_{0}^{*}$ theory with the central charges

$$
a_{H_{0}^{*}}=\frac{263}{768} \simeq 0.3424, \quad c_{H_{0}^{*}}=\frac{271}{768} \simeq 0.3529 .
$$

The $H_{0}^{*}$ SCFT was studied in Refs. [12,13] as a deformation of the $H_{0}$ Argyres-Douglas theory. Utilizing the UV Lagrangian description presented here, we are able to confirm various predictions about $H_{0}^{*}$.

Classically, the $F$ terms of Eq. (6) imply that $M, X$, and $O^{\prime} \equiv \operatorname{Tr} q \tilde{q}$ generate the chiral ring, with relations $M^{2} \sim 0$ and $O^{\prime 2} \sim 0$. The superconformal index for the $H_{0}^{*}$ theory can be computed to give a reduced index

$$
\mathcal{I}_{r}(t, y)=t^{3}-t^{7 / 2} \chi_{2}(y)+t^{4}+t^{7}+t^{17 / 2}+\cdots .
$$

From this, we see that the two generators $M$ and $O$ contribute the $t^{3}$ and $t^{4}$, respectively, while $X$ is not a generator. We also find that the operator $O_{\alpha}=\operatorname{Tr}\left(\phi W_{\alpha}\right)$ contributes to $t^{7 / 2} \chi_{2}(y)$. From the coefficients of $t^{6}, t^{7}, t^{8}$, we find that $M^{2}=M O^{\prime}=O^{\prime 2}=0$ in the chiral ring. The term $t^{7}$ comes from $\left(O_{\alpha}\right)^{2}$. There is a relation for $O_{\alpha}$ of the form $M O_{\alpha}=O^{\prime} O_{\alpha}=0$ which can be read from the absence of the terms $-t^{13 / 2} \chi_{2}(y)$ and $-t^{15 / 2} \chi_{2}(y)$. These relations support the analysis of Refs. $[12,13]$.

$H_{1}^{*}$ : Minimal a with $S U(2)$.- The flow to $H_{1}$ in our setup is a simplified version of the flow considered in Ref. [16], and it was also considered in Ref. [31]. From $H_{1}$, the $H_{1}^{*}$ SCFT is then obtained via a mass deformation to the singlet,

$$
W_{H_{1}^{*}}=X \operatorname{Tr} \phi^{2}+M \operatorname{Tr} q \tilde{q}+M^{2} .
$$

The central charges are

$a_{H_{1}^{*}}=\frac{927}{2048} \simeq 0.4526, \quad c_{H_{1}^{*}}=\frac{1023}{2048} \simeq 0.4995$.

Classically, the $F$ terms imply that the chiral ring is generated by $M, X, O_{2} \equiv \operatorname{Tr} \phi q q, O_{0} \equiv \operatorname{Tr} \phi q \tilde{q}, O_{-2} \equiv$ $\operatorname{Tr} \phi \tilde{q} \tilde{q}$, with relations $M^{2}=M O_{i}=X O_{i}=0$, and
$\mathrm{O}_{0}^{2} \sim \mathrm{O}_{2} \mathrm{O}_{-2}$. The last relation descends from that of the Higgs branch of the $H_{1}$ theory.

The reduced index is

$$
\begin{aligned}
\mathcal{I}_{r}(t, y ; a)= & t^{3}+t^{15 / 4}\left[\chi_{3}(a)-\chi_{2}(y)\right]+t^{9 / 2} \\
& -t^{6} \chi_{3}(a)+t^{15 / 2}\left[1+\chi_{5}(a)\right]+t^{33 / 4}+\cdots .
\end{aligned}
$$

We see that the theory has the $S U(2)$ current from the $-t^{6} \chi_{3}(a)$ term, which is visible at the level of the superpotential. There are generators $M, X$, and $O_{i}$ satisfying the relations $M^{2}=X^{2}=0$ and $O_{0}^{2} \sim O_{2} O_{-2}$. There are also fermionic operators $O_{\alpha}=\operatorname{Tr}\left(\phi W_{\alpha}\right)$ with relations $M O_{\alpha}=X O_{\alpha}=0$.

$\mathcal{T}_{\mu}$ : Next to minimal.-The $\mathcal{T}_{\mu}$ SCFT is obtained by the superpotential

$$
\begin{aligned}
W= & X \operatorname{Tr} \phi^{2}+M_{2} \operatorname{Tr} q \tilde{q}+M_{2}^{2} \\
& +M_{1} \operatorname{Tr} \phi q \tilde{q}+M_{3} \operatorname{Tr} \phi q q+M_{1} M_{3},
\end{aligned}
$$

and the central charges are given by

$a_{\mathcal{T}_{\mu}}=\frac{711}{2048} \simeq 0.3472, \quad c_{\mathcal{T}_{\mu}}=\frac{807}{2048} \simeq 0.3940$.

The value of $a$ is the third smallest value among the "good" theories that we find, and the second among the ones without flavor symmetry. The reduced index is

$$
\begin{aligned}
\mathcal{I}_{r}(t, y)= & t^{9 / 4}+t^{3}+t^{15 / 4}\left[1-\chi_{2}(y)\right]+t^{9 / 2}+t^{21 / 4} \\
& +t^{15 / 2}+t^{33 / 4} \chi_{2}(y)-t^{9} \chi_{3}(y)+\cdots .
\end{aligned}
$$

We see the chiral ring relations $\left(M_{1}\right)^{3}=\left(M_{2}\right)^{2}=\left(M_{3}\right)^{2}=0$. There is also an operator $O_{\alpha}=\operatorname{Tr}\left(\phi W_{\alpha}\right)$ with a relation $M_{2,3} O_{\alpha}=0$. There is no flavor symmetry, as we do not have the $-t^{6}$ term or a marginal operator.

$\mathcal{T}_{M}:$ A new minimal theory?.-Let us discuss one example among the 30 bad candidate fixed points. Consider the superpotential

$W=X \operatorname{Tr} \phi^{2}+M \operatorname{Tr} q \tilde{q}+M^{2}+\operatorname{Tr} \phi q q+\hat{X} \operatorname{Tr} \phi \tilde{q} \tilde{q}$.

There is no anomaly-free flavor symmetry in the Lagrangian. Assuming that the $R$ charges are fixed by $W$ and the anomaly condition, we get the central charges as

$a_{\mathcal{T}_{M}}=\frac{417}{2048} \simeq 0.2036, \quad c_{\mathcal{T}_{M}}=\frac{449}{2048} \simeq 0.2192$,

and also the superconformal index of this theory as

$$
\begin{aligned}
\mathcal{I}_{r}(t, y ; a)= & t^{3}-t^{15 / 4} \chi_{2}(y)+t^{9 / 2}+t^{21 / 4} \chi_{2}(y) \\
& -t^{6}+t^{15 / 2}-t^{33 / 4} \chi_{2}(y)+\cdots .
\end{aligned}
$$

The term $-t^{6}$ implies that there is a conserved current at the fixed point if it exists. The term $t^{21 / 4} \chi_{2}(y)$ violates the unitarity bound. As far as we know, this phenomenon has 
not been discussed in the literature. One possibility is that this term comes from the short multiplet $\overline{\mathcal{C}}_{-(1 / 4)(0,1 / 2)}$ (in the notation of Refs. [22,32,33]) that becomes free and gets decoupled along the RG flow. Subtracting the contribution to the central charges by treating the bottom component as a free fermion with $R=-1 / 4$, we get the central charges $(a, c)=\left(\frac{189}{1024}, \frac{189}{1024}\right) \simeq(0.1846,0.1846)$. We do not know if this prescription yields the correct central charges or index of the IR theory.

Even though we do not have a valid index, if we take it literally, we can read off the chiral ring relations $M^{2}=0$, $M \tilde{X}=0$, where $\tilde{X}$ is some combination of $X$ and $\hat{X}$. The other component is gone from the chiral ring.

Discussion.-One goal of this program is to search for and study minimal $\mathcal{N}=1$ SCFTs. One feature of the lowcentral charge SCFTs that we have examined here is that there is a chiral operator satisfying a relation of the form $O^{n} \sim 0$ for $n=2,3$. Another feature is that the central charges of the SCFTs considered here lie in a narrow range of $a / c$. It would be interesting to pursue the reasons for this, and search for other $\mathcal{N}=1$ SCFTs with truncated chiral rings.

A common property of the RG flows in this landscape is that some operators that are irrelevant at high energy can be relevant in the IR-such operators are called dangerously irrelevant. As such, this is an interesting arena for studying RG flows along the lines of Ref. [34].

At present, the status of the 36 "bad" theories is unclear because it is not clear how to account for the accidental symmetry in the $a$-maximization procedure and thus check if the corrected theory would flow to an interacting SCFT. One way forward would be to identify the fermionic multiplet that contributes to the unitary-violating terms in the index and decouple it, as we naively did for the $\mathcal{T}_{M}$ theory. It would be interesting to resolve this question and understand how the accidental symmetry arises. This would settle whether one of these theories is indeed a new candidate minimal $\mathcal{N}=1$ theory or strengthen the case for minimality of the $H_{0}^{*}$ theory.

We would like to thank Prarit Agarwal, Matthew Buican, Sergio Benvenuti, Stefano Cremonesi, Thomas Dumitrescu, Ken Intriligator, Kimyeong Lee, Sungjay Lee, Aneesh Manohar, Yu Nakayama, Yuji Tachikawa, and Piljin Yi for the helpful discussions. J. S. would like to thank the UCSD High Energy Theory group for hospitality. The work of K. M. was supported by JSPS KAKENHI Grant No. JP17K14296. The work of E. N. was supported in part by U.S. DOE Grant No. DE-SC0009919, and by a UC President's Dissertation Year Fellowship. The work of J. S. was supported in part by Overseas Research Program for Young Scientists through the Korea Institute for Advanced Study (KIAS). Part of this work was performed at the Aspen Center for Physics, which is supported by National Science Foundation Grant No. PHY-1607611. *maruyoshi@st.seikei.ac.jp

†enardoni@ucla.edu

\#jong@kias.re.kr

[1] J. L. Cardy, Phys. Lett. B 215, 749 (1988)

[2] Z. Komargodski and A. Schwimmer, J. High Energy Phys. 12 (2011) 099.

[3] R. Rattazzi, V. S. Rychkov, E. Tonni, and A. Vichi, J. High Energy Phys. 12 (2008) 031.

[4] D. Anselmi, D. Z. Freedman, M. T. Grisaru, and A. A. Johansen, Nucl. Phys. B526, 543 (1998).

[5] K. A. Intriligator and B. Wecht, Nucl. Phys. B667, 183 (2003).

[6] P. Liendo, I. Ramirez, and J. Seo, J. High Energy Phys. 02 (2016) 019.

[7] P. C. Argyres and M. R. Douglas, Nucl. Phys. B448, 93 (1995).

[8] P. C. Argyres, M. R. Plesser, N. Seiberg, and E. Witten, Nucl. Phys. B461, 71 (1996).

[9] D. Poland, D. Simmons-Duffin, and A. Vichi, J. High Energy Phys. 05 (2012) 110.

[10] N. Bobev, S. El-Showk, D. Mazac, and M. F. Paulos, J. High Energy Phys. 08 (2015) 142.

[11] D. Poland and A. Stergiou, J. High Energy Phys. 12 (2015) 121.

[12] D. Xie and K. Yonekura, Phys. Rev. Lett. 117, 011604 (2016).

[13] M. Buican and T. Nishinaka, Phys. Rev. D 94, 125002 (2016).

[14] See also a recent work on 3D $\mathcal{N}=4$ theory [D. Gang and M. Yamazaki, Phys. Rev. D 98, 121701 (2018)].

[15] K. Maruyoshi and J. Song, Phys. Rev. Lett. 118, 151602 (2017).

[16] K. Maruyoshi and J. Song, J. High Energy Phys. 02 (2017) 075.

[17] P. Agarwal, K. Maruyoshi, and J. Song, J. High Energy Phys. 12 (2016) 103.

[18] J. Kinney, J. M. Maldacena, S. Minwalla, and S. Raju, Commun. Math. Phys. 275, 209 (2007).

[19] C. Romelsberger, Nucl. Phys. B747, 329 (2006).

[20] D. M. Hofman and J. Maldacena, J. High Energy Phys. 05 (2008) 012.

[21] D. Kutasov, A. Parnachev, and D. A. Sahakyan, J. High Energy Phys. 11 (2003) 013.

[22] C. Beem and A. Gadde, J. High Energy Phys. 04 (2014) 036.

[23] M. Evtikhiev, J. High Energy Phys. 04 (2018) 120.

[24] K. A. Intriligator and N. Seiberg, Nucl. Phys. B431, 551 (1994).

[25] S. Elitzur, A. Forge, A. Giveon, and E. Rabinovici, Nucl. Phys. B459, 160 (1996).

[26] See Supplemental Material at http://link.aps.org/ supplemental/10.1103/PhysRevLett.122.121601 for the full list of superconformal fixed points.

[27] N. Bobev and P. M. Crichigno, J. High Energy Phys. 12 (2017) 065.

[28] There are two theories with three conserved currents with smaller $a$, but we do not find any evidence for the $S U(2)$ symmetry.

[29] The theory with smaller $c$ than $H_{0}^{*}$ was also noticed by Sergio Benvenuti. We thank him for informing us about this. 
[30] We might have anticipated this from the fact that $X$ was introduced to decouple $\operatorname{Tr} \phi^{2}$, but as we see in later examples, $X$ might remain a generator.

[31] S. Benvenuti and S. Giacomelli, Phys. Rev. Lett. 119, 251601 (2017).
[32] F. A. Dolan and H. Osborn, Ann. Phys. (Amsterdam) 307, 41 (2003).

[33] A. Gadde, L. Rastelli, S. S. Razamat, and W. Yan, J. High Energy Phys. 03 (2011) 041.

[34] S. Gukov, J. High Energy Phys. 01 (2016) 020. 